\title{
openheart Characterisation of clot microstructure properties in stable coronary artery disease
}

Ahmed Sabra, ${ }^{1,2,3}$ Matthew James Lawrence, ${ }^{1,2}$ Robert Aubrey, ${ }^{1}$ Daniel Obaid, ${ }^{4}$ Alexander Chase, ${ }^{4}$ Dave Smith, ${ }^{4}$ Phillip Thomas, ${ }^{4}$ Sharon Storton, ${ }^{1,2}$ Gareth R Davies, ${ }^{1,2}$ Karl Hawkins, ${ }^{2}$ Phylip Rhodri Williams, ${ }^{5}$ Keith Morris, ${ }^{6}$ Phillip Adrian Evans ${ }^{1,2,7}$

To cite: Sabra A, Lawrence MJ, Aubrey R, et al. Characterisation of clot microstructure properties in stable coronary artery disease. Open Heart 2017;4:e000562. doi:10.1136/ openhrt-2016-000562

AS and MJL contributed equally.

Received 8 November 2016 Revised 20 February 2017 Accepted 4 April 2017

\section{CrossMark}

${ }^{1}$ NISCHR Haemostasis Biomedical Research Unit, Morriston Hospital, ABMU Health Board, Swansea, UK ${ }^{2}$ NISCHR Haemostasis Biomedical Research Unit, College of Medicine, Swansea University, Swansea, UK ${ }^{3}$ Department of Cardiology, Princess of Wales Hospital, ABMU Health Board, Bridgend, UK

${ }^{4}$ Cardiac Centre, Morriston Hospital, ABMU Health Board, Swansea, UK

${ }^{5}$ College of Engineering, Swansea University, Swansea, UK

${ }^{6}$ School of Applied Science, Cardiff Metropolitan University, Cardiff, UK

${ }^{7}$ Department of Emergency Medicine, Morriston Hospital, ABMU Health Board, Swansea, UK

Correspondence to Phillip Adrian Evans; phillip. evans2@wales.nhs.uk

\section{ABSTRACT}

Background Coronary artery disease (CAD) is associated with an increased prothrombotic tendency and is also linked to unfavourably altered clot microstructure. We have previously described a biomarker of clot microstructure $\left(d_{f}\right)$ that is unfavourably altered in acute myocardial infarction. The $d_{f}$ biomarker assesses whether the blood will form denser or looser microstructures when it clots. In this study we assessed in patients with stable chest pain whether $d_{f}$ can differentiate between obstructed and unobstructed CAD.

Methods A blood sample prior to angiography was obtained from 251 consecutive patients undergoing diagnostic coronary angiography. Patients were categorised based on angiographic findings as presence or absence of obstructive CAD (stenosis $\geq 50 \%$ ). The blood sample was assessed using the $d_{f}$ biomarker, standard laboratory markers and platelet aggregometry (Multiplate). Results A significant difference $(p=0.028)$ in $d_{f}$ was observed between obstructive CAD $(1.748 \pm 0.057$, $\mathrm{n}=83)$ and unobstructive CAD $(1.732 \pm 0.052, \mathrm{n}=168)$, where patients with significant CAD produce denser, more tightly packed clots. $d_{f}$ was also raised in men with obstructive CAD compared with women $(1.745 \pm 0.055$ vs $1.723 \pm 0.052, p=0.007$ ). Additionally $d_{f}$ significantly correlated with the platelets response to arachidonic acid as measured by the ASPItest area under the curve readings from platelet aggregometry (correlation coefficient=0.166, $p=0.008$ ), a low value of the ASPItest indicating effective aspirin use was associated with looser, less dense clots.

Conclusions For the first time, we characterise clot microstructure, as measured by $d_{f}$, in patients with stable CAD. $d_{f}$ can potentially be used to risk-stratify patients with stable CAD and assess the efficacy of therapeutic interventions by measuring changes in clot microstructure.

\section{INTRODUCTION}

Coronary artery disease (CAD) is associated with an underlying systemic imbalance in haemostasis caused by the presence of a hypercoagulable state and a decrease in fibrinolysis. ${ }^{1-4}$ While CAD has been linked to an increased prothrombotic state, no marker has been identified that can accurately assess

\section{KEY QUESTIONS}

What is already known about this subject? Coronary artery disease (CAD) alters coagulation and is associated with an increased risk of thrombotic disease.

What does this study add?

We show how a novel marker of clot microstructure can be used to characterise the level of disease in stable CAD and therapeutic manipulation.

How might this impact on clinical practice? We identify a possible tool for risk-stratifying patients with stable CAD, alongside the potential to assess the efficacy of therapeutic interventions.

abnormalities of global haemostasis due to this process and to severity of disease. Identifying a global haemostatic marker of coagulability and fibrinolysis may be important in stratifying risk of atherothrombosis and providing the basis for individualised therapeutic management.

Previous studies have identified that abnormal clot microstructure is of significant importance in the pathophysiology of many vascular and inflammatory disease states including CAD. ${ }^{5-8}$ However, the standard techniques for assessing clot microstructure do not translate to being used as routine markers in a clinical setting. ${ }^{9}$ This has led to the development of a technique that uses assessment of the viscoelastic properties of coagulating blood to quantify its clot microstructure as a fractal dimension, $\mathrm{d}_{\mathrm{f}}{ }^{9} \mathrm{In}$ contrast to standard coagulation assays, the $d_{r}$ measurement is performed using unadulterated whole blood in a near patient setting and provides rapid assessment of coagulation. Lower values of $d_{f}$ correspond to less dense, less branched, weaker clots, whereas higher $d_{f}$ values represent denser, more complex, 
stronger clots. ${ }^{9}$ The $\mathrm{d}_{\mathrm{f}}$ measurement has been previously validated in several disease states and has also been used to stratify the severity of disease, however, its role in stable CAD remains unclear. ${ }^{10-14}$

The aim of the present study was to characterise clot microstructure in CAD. The hypothesis was that for a cohort of patients with suspected CAD undergoing diagnostic angiography, $d_{f}$ will be unfavourably altered in those patients with obstructive CAD compared with those with no or unobstructive CAD.

\section{METHODS}

\section{Patient population}

This study was conducted in accordance with good clinical practice and has been reviewed and approved by the by the local Research Ethics Committee (Wales REC 7). We screened all consecutive patients undergoing routine diagnostic coronary angiography for evaluation of new onset chest pain, who have no previously confirmed CAD. Eligible patients were recruited from two hospitals in South Wales (a large teaching hospital and a district general hospital) from November 2012 to August 2014. We excluded patients with active cancer; liver disease; chronic kidney disease stage IV and V or on dialysis; $<18$ years of age; known clotting disorders; history of myocardial infarction, stroke or thromboembolic disease; severe heart failure (ejection fraction $<35 \%$ or clinically New York Heart Association (NYHA) stage III-IV) or taking anticoagulants at the time of the study. Written informed consent was obtained from all patients before recruitment in the study.

One venous blood sample was collected before angiography. Data including demographics, medical history and current medications were collected for each patient, including presence of diabetes, family history of CAD (history of acute myocardial in a first-degree relative), hypercholesterolaemia (total cholesterol $>5 \mathrm{mmol} / \mathrm{L}$ or currently on medication for high cholesterol) and smoking history.

Patients were divided in two groups based on coronary angiographic findings: those with angiographically normal arteries or minor irregularities but no significant stenosis $(\geq 50 \%)$ were termed unobstructed, those with any coronary stenosis $\geq 50 \%$ being defined as obstructive CAD. Clinicians reporting coronary angiography findings were blinded to the results of biomarker analysis and the operator performing biomarker analysis was blinded to the angiography results.

\section{Blood sampling}

Each blood sample was divided into several aliquots. One aliquot of whole venous blood was immediately transferred and used for viscoelastic measurements. The remaining aliquots were used to perform standard coagulation screens, full blood count, thrombin generation or platelet aggregometry (see below).

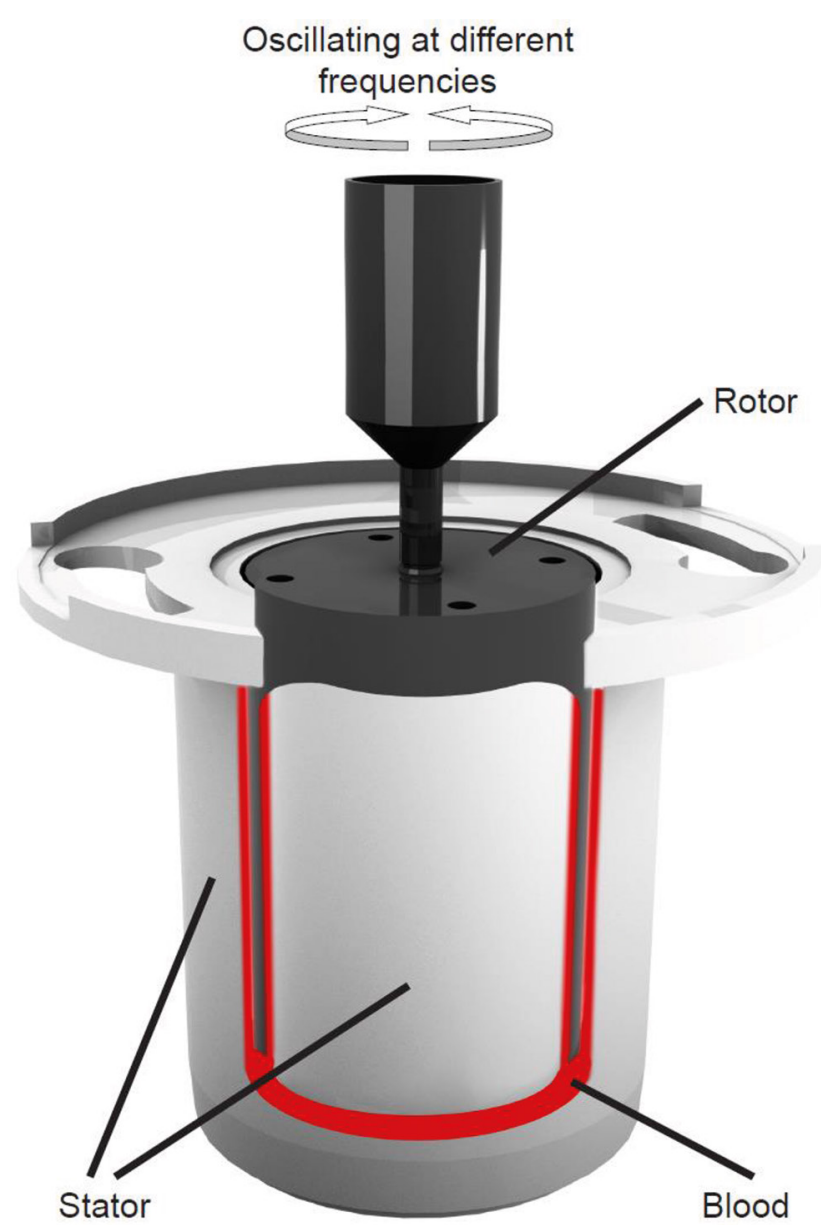

Figure 1 Diagram of a double-gap concentric cylinder measurement geometry. The double-gap geometry consists of a stationary cup or stator into which a $6.6 \mathrm{~mL}$ sample of blood is added after which a bob that is free to rotate called a rotor is then lowered into the sample. The movement of the rotor is controlled by an AR-G2-controlled stress rheometer and will oscillate at four different frequencies $(0.20,0.43,0.93$ and $2.00 \mathrm{~Hz}$ ) sequentially over time.

\section{Viscoelastic measurements}

The viscoelastic measurements are based on attainment of the gel point (GP) from which the fractal dimension, $\mathrm{d}_{\mathrm{f}}$, is determined. ${ }^{9}$ The GP technique has been previously validated for use with blood in several studies. ${ }^{10-15}$ Briefly, blood is placed within the double concentric measuring geometry of a controlled stress rheometer, AR-G2 (TA Instruments, New Castle, DE, USA) which is held a constant temperature of $37^{\circ} \mathrm{C} \pm 0.1^{\circ} \mathrm{C}$ (figure 1). Immediately after loading the blood into the AR-G2, viscoelastic analysis is preformed using small amplitude oscillatory shear measurements at varying frequencies; $2,0.93,0.43$ and $0.2 \mathrm{~Hz}$, with an applied peak stress amplitude of 0.03 $\mathrm{Pa}$. Repeatedly performing these measurements over time allows for the measurement of the GP (figure 2). The GP marks the transition of the blood from a viscoelastic liquid to a viscoelastic solid, where the GP identifies the formation of the incipient blood clot or the first point which a sample spanning (haemostatic) structure can be 
identified. ${ }^{9}$ In figure 2 the GP is located when the four frequencies cross-over. From the GP measurement we can quantify how the fibrin clot is organised by calculating its corresponding fractal dimension, $\mathrm{d}_{\mathrm{f}}{ }^{9}$

\section{Laboratory markers}

A $4 \mathrm{~mL}$ aliquot of blood was drawn into tubes containing EDTA for Full Blood Count (FBC) analysis and then analysed using a Sysmex XE 2100 (Sysmex UK, Milton Keynes, UK). Parameters measured included: haemoglobin, haematocrit and platelet count. A $4.5 \mathrm{~mL}$ aliquot was collected into tubes containing citrate and then analysed using a Sysmex CA1500 (Sysmex UK, Milton Keynes, UK). Parameters measured included: prothrombin time, activated partial thromboplastin time, factor VIII and Clauss fibrinogen. D-dimer analysis was carried out using a latex immunoturbidimetric assay Hemosil HS D-dimer (Instrumentation Laboratory, Warrington, UK) with a ACL TOP 500 (Instrumentation Laboratory, Warrington, UK). Plasma cytokines (interleukin 6 and myeloperoxidase) were measured and quantified using a standard ELISA (Quantikine, R+D Systems, UK), according to the manufacturer's instructions.

\section{Thrombin generation}

Thrombin generation was measured using the Thrombin Generation Assay (TGA, Technoclone Diagnostics, Vienna, Austria). Plates were loaded into the fluorogenic plate reader TECAN infinite F200 pro (Labtech International, Uckfield, UK) and read every $60 \mathrm{~s}$ for 1 hour. TGA software was used to calculate individual thrombin generation curves.

\section{Platelet aggregation measurements}

Measurement of platelet aggregation was achieved using the Multiplate analyser (Dynabyte $\mathrm{GmBH}$, Munich, Germany). An aliquot of whole blood $(3 \mathrm{~mL})$ was transferred to hirudin tubes (Roche Diagnostics $\mathrm{GmbH}$, Mannheim, Austria, Ref: 06675751) and kept at room temperature for $30 \mathrm{~min}$ before testing. Three hundred microlitres of whole hirudinated blood was added to $300 \mu \mathrm{L}$ of saline preheated to $37^{\circ} \mathrm{C}$ and allowed to incubate for $3 \mathrm{~min}$ in individual test cells. Following incubation platelet activation was induced by addition of specific agonists to respective test cells, and electrical impedance was recorded. The agonists included ADP $(20 \mu \mathrm{L}$ of $0.2 \mathrm{mM}$ stock solution) for measuring P2Y12 receptor aggregation, which is inhibited by clopidogrel and other thienopyridines. The second agonist was ASPItest reagent ( $20 \mu \mathrm{L}$ of $15 \mathrm{mM}$ stock solution) for measuring the inhibitory effect of aspirin.

\section{Statistical analysis}

A power calculation was performed assuming a mean difference in $d_{f}$ of 0.025 (based on pilot data) between unobstructive and obstructive CAD. Taking a SD of 0.045 , a power of 0.85 and significance value set at $0.05 \mathrm{a}$ minimum of 65 patients in both groups is required. With the study designed for consecutive patients and with a likely recruitment bias towards unobstructive CAD, we aimed to recruit double that number. Descriptive analyses were performed to establish baseline characteristics for both groups. Categorical variables are summarised using percentages and compared using $\chi^{2}$ tests while continuous variables are presented using mean and SD unless otherwise stated. Differences between groups were compared using two sample t-tests for parametric data or Kruskal-Wallis test for non-parametric data. Pearson correlation was undertaken to explore associations between $d_{f}$ and demographic, laboratory markers and platelet aggregometry. Statistical analysis was performed using Minitab V.15 software (Havertown, Pennsylvania, USA) and deemed significant when $\mathrm{p}<0.05$.

\section{RESULTS}

A total of 275 patients were recruited, full angiographic, viscoelastic measurements and platelet aggregation measurements were performed successfully in 251. Of the 251 patients recruited, 168 patients were classed as unobstructed $\mathrm{CAD}$ and 83 patients as obstructive CAD. The baseline characteristics and patient demographics for the two groups is recorded in table 1. Significant differences between the demographics of the two groups are observed for age $(\mathrm{p}=0.013)$, sex $(\mathrm{p}<0.001)$ and statin use $(p=0.045)$. Results of the viscoelastic testing, laboratory markers and platelet aggregometry measurements can be found in table 2 .

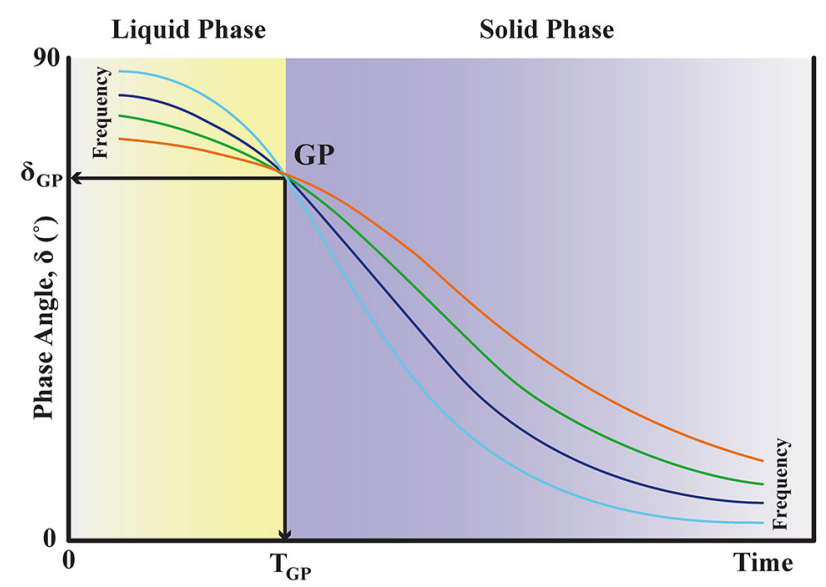

Figure 2 Gel point (GP) trace. This represents a typical GP result for one sample of blood. The illustration demonstrates how phase angle, $\delta$, changes as coagulation progresses. $\delta$ has a range of $0^{\circ}$ to $90^{\circ}$, where $90^{\circ}$ identifies a purely viscous response and $0^{\circ}$ identifies a purely elastic response with any value in between being a measure of the viscoelastic response to imposed stress. In a material that is changing from a liquid to a solid such as blood, there will be a decrease in $\delta$. At the establishment of the incipient clot, when the clot becomes a viscoelastic solid, there is a point where the value of $\delta$ will be independent of frequency called the GP. The structural property of the incipient clot (in terms of its fractal dimension, $\mathrm{d}_{\mathrm{f}}$ ) is derived from this frequency independent value of $\delta_{\mathrm{GP}}$. 
Table 1 Patient baseline characteristics and demographics

\begin{tabular}{|c|c|c|c|c|c|}
\hline & Normal $(n=80)$ & $\begin{array}{l}\text { Unobstructed CAD } \\
(\mathrm{n}=88)\end{array}$ & Unobstructed $(n=168)$ & Obstructive CAD $(n=83)$ & p Value \\
\hline Age & $58.6 \pm 8.8$ & $63.5 \pm 10.3$ & $61.1 \pm 9.8$ & $64.3 \pm 9.7$ & 0.013 \\
\hline Sex $(M: W)$ & $32: 48(40 \%)$ & $45: 43(51 \%)$ & $77: 91(46 \%)$ & $58: 25(70 \%)$ & $<0.001$ \\
\hline BMI & $30.3 \pm 5.4$ & $30.8 \pm 5.7$ & $30.4 \pm 5.5$ & $30.0 \pm 7.0$ & 0.59 \\
\hline Smoking (current) & $10(12 \%)$ & $14(16 \%)$ & $24(14 \%)$ & $14(17 \%)$ & 0.34 \\
\hline Hypercholesterol & $54(68 \%)$ & $71(81 \%)$ & $125(74 \%)$ & $69(83 \%)$ & 0.31 \\
\hline HTN & $40(50 \%)$ & $40(45 \%)$ & $90(54 \%)$ & $43(52 \%)$ & 0.82 \\
\hline DM & $12(15 \%)$ & $29(33 \%)$ & $41(24 \%)$ & $16(19 \%)$ & 0.19 \\
\hline $\mathrm{FHx} \mathrm{Ml}$ & $33(42 \%)$ & $47(53 \%)$ & $80(48 \%)$ & $39(47 \%)$ & 0.91 \\
\hline Antiplatelet use & $60(75 \%)$ & $51(58 \%)$ & $111(66 \%)$ & $66(78 \%)$ & 0.11 \\
\hline Aspirin & 58 & 34 & 92 & 57 & \\
\hline P2Y12 & 0 & 6 & 6 & 2 & \\
\hline Both & 2 & 11 & 13 & 7 & \\
\hline Statins & $50(63 \%)$ & $50(63 \%)$ & $109(65 \%)$ & $66(80 \%)$ & 0.045 \\
\hline
\end{tabular}

All Caucasians but two. Other lowering lipid drugs: four ezetimibe and two fenofibrates.

$\mathrm{BMI}$, body mass index; CAD, coronary artery disease; DM, diabetes mellitus; FHx MI, family history of stroke myocardial infarction; HTN,

hypertension; M:W, men:women.

\section{Viscoelastic measurements}

A significant increase in the value of $d_{f}$ was observed for those patients with obstructive CAD when compared with unobstructed $\left(\mathrm{d}_{\mathrm{f}}=1.748 \pm 0.057\right.$ vs $1.732 \pm 0.052$, $\mathrm{p}=0.028$ ). We also preformed an analysis of covariance and analysis using a general linear model. This analysis demonstrated the difference in $d_{f}$ between obstructive
$\mathrm{CAD}$ and the unobstructed group remains significant $(p<0.05)$ even when we adjust for fibrinogen concentration, haematocrit, antiplatelet function or the presence of hypercholesterolaemia. Furthermore, we analysed the data by dividing the unobstructed group into two separate groups, normal $(n=80)$ and unobstructed CAD $(n=88)$ giving total of three groups. Using a one-way analysis

Table 2 Results of the viscoelastic testing, standard and specific markers for non-severe and severe CAD groups

\begin{tabular}{|c|c|c|c|c|c|}
\hline & Normal $(n=80)$ & $\begin{array}{l}\text { Unobstructed CAD } \\
(n=88)\end{array}$ & Unobstructed $(n=168)$ & Obstructive CAD ( $n=83)$ & p Value \\
\hline$d_{f}$ & $1.728 \pm 0.052$ & $1.735 \pm 0.053$ & $1.732 \pm 0.052$ & $1.749 \pm 0.057$ & 0.028 \\
\hline IL-6 & $50.1 \pm 5.0$ & $52.1 \pm 4.3$ & $51.6 \pm 4.1$ & $67.6 \pm 7.5$ & 0.38 \\
\hline MPO & $4541 \pm 1450$ & $4563 \pm 1471$ & $4544 \pm 1456$ & $4636 \pm 1482$ & 0.66 \\
\hline CRP & $3.6 \pm 3.2$ & $3.7 \pm 3.0$ & $3.7 \pm 2.8$ & $3.7 \pm 3.0$ & 0.75 \\
\hline $\mathrm{Hb}(\mathrm{g} / \mathrm{dL})$ & $14.0 \pm 1.2$ & $14.3 \pm 1.3$ & $14.1 \pm 1.3$ & $14.2 \pm 1.2$ & 0.45 \\
\hline PIt $\left(\times 10^{9} / L\right)$ & $263 \pm 67$ & $253 \pm 69$ & $258 \pm 67$ & $249 \pm 53$ & 0.22 \\
\hline HCT $(g / g)$ & $0.418 \pm 0.033$ & $0.425 \pm 0.035$ & $0.422 \pm 0.035$ & $0.437 \pm 0.035$ & 0.24 \\
\hline PT (s) & $10.5 \pm 0.4$ & $10.5 \pm 0.5$ & $10.5 \pm 0.4$ & $10.6 \pm 0.5$ & 0.057 \\
\hline APTT (s) & $25.8 \pm 2.1$ & $25.8 \pm 1.9$ & $25.8 \pm 2.0$ & $26.2 \pm 1.9$ & 0.13 \\
\hline $\mathrm{FBG}(\mathrm{g} / \mathrm{L})$ & $3.3 \pm 0.6$ & $3.4 \pm 0.7$ & $3.3 \pm 0.6$ & $3.4 \pm 0.7$ & 0.26 \\
\hline $\mathrm{DD}^{*}$ & 94 (IQR 71-147) & 122 (IQR 81-178) & 110 (IQR 74-167) & 131 (IQR 80-209) & 0.095 \\
\hline $\mathrm{TG}$ & $137 \pm 44$ & $119 \pm 50$ & $128 \pm 76$ & $136 \pm 93$ & 0.51 \\
\hline FVIII & $132 \pm 44$ & $138 \pm 46$ & $135 \pm 45$ & $135 \pm 38$ & 0.99 \\
\hline Multiplate ADP & $85.3 \pm 32.2$ & $77.1 \pm 27.5$ & $81.4 \pm 29.8$ & $76.9 \pm 28.5$ & 0.24 \\
\hline Multiplate ASPI* & 28 (IQR 13-70) & 30 (IQR 18-83) & 29.0 (IQR 15.8-78.1) & 25.4 (IQR 18.0-64.5) & 0.41 \\
\hline
\end{tabular}

*Median and interquartile values reported.

APPT, activated partial thromboplastin time; CAD, coronary artery disease; CRP, C-reactive protein; DD, D-Dimer; FBG, fibrinogen; FVIII, Ffactor VIII; Hb, haemoglobin; HCT, haematocrit; IL-6, interleukin 6; MPO, myeloperoxidase; PIt, platelet count; PT, prothrombin time; TG, thrombin generation. 


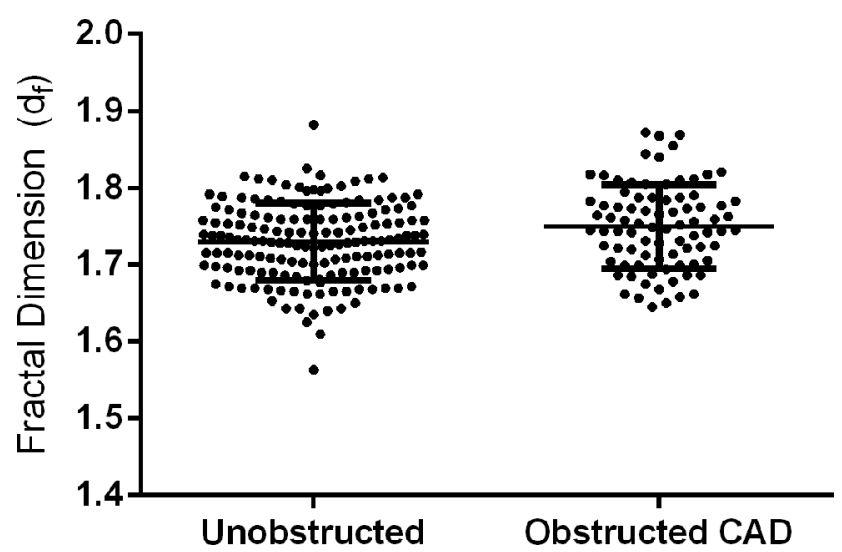

Figure 3 A graph showing the dispersion of $d_{f}$ measurements among the unobstructed and the obstructed coronary artery disease (CAD) groups, where a significant increase in the value of $d_{f}$ was observed for those patients with obstructive CAD when compared with unobstructed $\left(d_{f}=1.748 \pm 0.057\right.$ vs $\left.1.732 \pm 0.052, p=0.028\right)$.

of variance (ANOVA; 95\% CI) we observe a non-significant $(\mathrm{p}=0.053)$ increase in $\mathrm{d}_{\mathrm{f}}$ when comparing the three groups: normal $\left(\mathrm{n}=80, \mathrm{~d}_{\mathrm{f}}=1.728 \pm 0.052\right)$; unobstructed CAD $(1 \%<$ stenosis $<50 \%) \quad\left(n=88, \quad d_{f}=1.735 \pm 0.053\right)$ and obstructed CAD ( $\geq 50 \%$ stenosis) $\left(n=83, d_{f}=1.748 \pm 0.057\right)$. In addition, we reviewed the obstructed CAD group and classified it into 1, 2 and 3 vessel disease to assess whether there was a difference in $d_{f}$. We found no significant difference in $\mathrm{d}_{\mathrm{f}}$ depending on number of diseased vessels $(p=0.83)\left(1\right.$ vessel $(n=40) d_{f}=1.738 \pm 0.060,2$ vessel $(n=27)$ $d_{f}=1.770 \pm 0.055$ and 3 vessel $\left.(n=14) \quad d_{f}=1.752 \pm 0.056\right)$. Dispersion of $\mathrm{d}_{\mathrm{f}}$ for both the unobstructed and obstructed CAD groups can be seen in figure 3 .

Comparing the $\mathrm{d}_{\mathrm{f}}$ against patient demographics, we only found significant differences in men versus women, where $\mathrm{d}_{\mathrm{f}}$ was raised in men $(1.745 \pm 0.055$ vs $1.723 \pm 0.052$, $\mathrm{p}=0.007)$, and in smokers versus non/ex-smokers, where $\mathrm{d}_{\mathrm{f}}$ was raised in smokers $(1.761 \pm 0.037$ vs $1.733 \pm 0.055$, $\mathrm{p}=0.001)$. The differences observed in smokers versus non/ex-smokers persisted when studying the obstructive $(1.754 \pm 0.050$ vs $1.728 \pm 0.054, \mathrm{p}=0.003)$ and unobstructive CAD (1.776 \pm 0.042 vs $1.745 \pm 0.059, \mathrm{p}=0.043)$ groups.

\section{Laboratory markers and thrombin generation}

We found no significant difference between unobstructed and obstructive CAD for any of the standard laboratory tests (see table 2). Positive correlations were observed between $\mathrm{d}_{\mathrm{f}}$ and two of the laboratory markers: haematocrit (correlation coefficient $=0.458, \mathrm{p}=0.001$ ) and fibrinogen concentration (correlation coefficient $=0.242$, $\mathrm{p}=0.001$ ), where these relationships persisted when studying all patients recruited or in their individual obstructive (fibrinogen concentration: 0.308, $\mathrm{p}=0.004$; haematocrit: $0.412, \mathrm{p}=0.001$ ) or unobstructive (fibrinogen concentration: $0.186, \mathrm{p}=0.016$; haematocrit: 0.477 , $\mathrm{p}=0.001)$ CAD groups. We also reanalysed the laboratory markers data using one-way ANOVA (95\% CI) to look at the data in three groups, namely normal, unobstructed
CAD and obstructed CAD, however, we found no differences between the three groups.

\section{Platelet aggregometry}

The use of antiplatelet therapy was only recorded when it was known to be effective in the patient. The effectiveness of antiplatelet therapy was determined from platelet aggregometry measurements taken at the same time as $\mathrm{d}_{\mathrm{f}}$ measurements. Effective aspirin use is recorded by an ASPI reading of below 40IUs as deemed in a previous publication. ${ }^{16}$ Effective inhibition of P2Y12 receptor on the platelet is determined by an ADP value of below 47IUs. ${ }^{17}$ We found that $70 \%$ of patients were receiving some form of antiplatelet therapy with $89 \%$ of those receiving monotherapy and $11 \%$ dual antiplatelet therapy. Of the monotherapy group 95\% were taking aspirin and 5\% were taking a P2Y12 inhibitor. A significant positive correlation was observed between ASPI and $\mathrm{d}_{\mathrm{f}}(0.157 \mathrm{p}=0.014)$ but not ADP $(0.089 \mathrm{p}=0.34)$. We found a non-significant increase in $\mathrm{d}_{\mathrm{f}}$ for those not taking antiplatelet therapy compared with those taking antiplatelet therapy $(1.735 \pm 0.053$ vs $1.746 \pm 0.053, \mathrm{p}=0.12)$. A significant reduction in $d_{f}$ was observed for patients on dual antiplatelet therapy compared with no therapy (1.722 \pm 0.053 vs $1.746 \pm 0.053, \mathrm{p}=0.048)$.

\section{DISCUSSION}

This study for the first time shows that a whole blood biomarker that quantifies clot microstructure can be used to discriminate between those with unobstructed and those with obstructive CAD. We show that patients with obstructive CAD produce clots with a significantly higher $\mathrm{d}_{\mathrm{f}}(1.748 \pm 0.057)$ when compared with those patients without obstructive CAD (1. 732 \pm 0.052$)(p=0.028)$ (see figure 3 ). An increased value of $d_{f}$ can be illustrated using a previously published computational model of a simplified branching network, which shows the relationship between $\mathrm{d}_{\mathrm{f}}$ and the mass of the network. ${ }^{1819}$ In figure 4 we show that a change in $\mathrm{d}_{\mathrm{f}}$ from 1.732 (unobstructed CAD) to 1.748 (obstructive CAD) would correspond to an additional $25 \%$ more mass that would need to be incorporated into the structure of the incipient clot. Consequently, patients with obstructive CAD are more likely to produce clot microstructures that are consistently denser and more tightly packed, resulting in a clot that is of poorer quality, harder to breakdown and more likely to form a thrombus. This may be linked to the underlying hypercoagulable state of CAD patients suggested in previous studies. $^{1220}$

While a difference in the value of $d_{f}$ is observed between the obstructive and unobstructive CAD groups, it is important to note that several patient demographics are also significantly different (see table 1 ). We found age was significantly different, where patients with unobstructed CAD were on average 3.2 years younger than the obstructive CAD group $(61.1+9.8$ years vs $64.3+9.7$ years, respectively, $\mathrm{p}=0.013)$. This is perhaps unsurprising as 


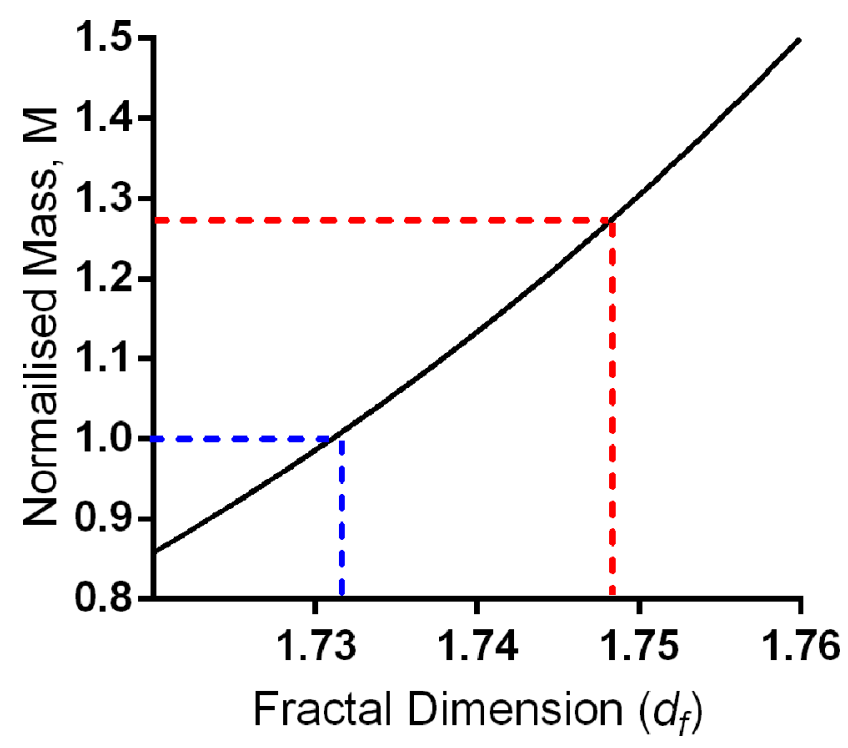

Figure 4 Fractal dimension $\left(d_{f}\right)$ versus mass. Graph showing the simplified non-linear relationship between $d_{f}$ and the amount of mass incorporated into the incipient clot microstructure. Fibrin networks have fractal properties, where the mass, $M$, is related to $d_{f}$ by the following power law equation $\left(M \approx \varepsilon d_{f}\right.$, where $\varepsilon$ is some length scale value in the range $100 \mathrm{~nm}$ to $10 \mu \mathrm{m})$. The mass value on the $\mathrm{y}$-axis is normalised to the unobstructed CAD value of $d_{f}=1.732$, where at $a_{\mathrm{f}}$ of 1.732 the normalised mass value is equal to 1 (blue dotted lines). The figure illustrates that a structure that forms with $a d_{f}$ of 1.748 , like the mean value of the obstructive CAD group (red dotted lines) would have an increased relative mass compared with a structure that formed with a $d_{f}$ value of 1.732 , having 1.27 times more mass.

age is a primary risk factor in advanced CAD. ${ }^{21}$ However, we found no significant correlations between age and $\mathrm{d}_{\mathrm{f}}$ either in all patients or in their individual groups (all patients: correlation coefficient $=-0.118, \mathrm{p}=0.064$; obstructive CAD: $-0.210, \mathrm{p}=0.055$; unobstructive CAD: -0.105 , $\mathrm{p}=0.164)$. Furthermore, in a previous study with a healthy control (70.6 6 7.1 years), there was no correlation between age and $d_{f}{ }^{11}$ The findings herein may be surprising considering studies have shown that increased age is often associated with an increase in certain haematological characteristics, such as fibrinogen concentration. ${ }^{22}$ Fibrinogen concentration has also been associated with cardiovascular risk; in the present study we did identify a positive correlation between $d_{f}$ and fibrinogen concentration but fibrinogen was not significantly raised when comparing obstructive and unobstructive CAD. ${ }^{23}$ This suggests that while age is associated with advanced CAD, age itself is not the driving force behind the development of abnormal clot formation.

Sex was also significantly different between obstructive and unobstructive $\mathrm{CAD}$, where there was a significantly higher proportion of men in the obstructive CAD group compared with unobstructed CAD group (70\% vs $46 \%$, $\mathrm{p}<0.001)$. This is unsurprising as CAD is often reported as more prevalent in men. ${ }^{21}$ When comparing $\mathrm{d}_{\mathrm{f}}$ versus sex, we found that $d_{f}$ was significantly increased in men compared with women $(1.746+0.055$ vs $1.727+0.052$, $\mathrm{p}=0.007)$. In the present study, the higher value of $d_{f}$ in men may be due to the increased number of men with obstructive CAD compared with women, however, the raised $d_{f}$ in men persists across both the obstructed $(1.753 \pm 0.059$ vs $1.737 \pm 0.053)$ and unobstructed $(1.740 \pm 0.059$ vs $1.725 \pm 0.053) \mathrm{CAD}$. Another rationale may be that men in general have an underlying propensity to form denser and more compact clots (hence higher $d_{\mathrm{f}} \mathrm{s}$ ) than women. However, a previous study which used a healthy control $(n=74)$ did not find a difference in the value of $d_{f}$ between men and women. ${ }^{11} \mathrm{~A}$ previous study investigating myocardial infarction with $d_{f}$ found that men were also more likely to have increased $\mathrm{d}_{\mathrm{f}}$ values compared with women. ${ }^{14}$ Men with $\mathrm{CAD}$ have been shown to have a higher prevalence of experiencing an acute coronary syndrome or fatal coronary heart disease. ${ }^{21} \mathrm{~A}$ final explanation may be that men with $\mathrm{CAD}$ (either obstructive or unobstructive) are more likely to form denser more compact clots (hence higher $\mathrm{d}_{\mathrm{f}} \mathrm{s}$ ) than women, thus are at an increased risk of suffering an acute coronary syndrome.

Statin use was also found to be different when comparing the obstructive and unobstructive CAD groups. Statin use was significantly increased in the obstructive CAD group ( $80 \%$ vs $65 \%, \mathrm{p}=0.045$ ) (table 2 ). With the increased mean age and percentage of men in the obstructive CAD group, it is unsurprising that statin use is raised with both being strong indicators for statin use. ${ }^{24}{ }^{25}$ A previous study has shown that statin use has a mediating effect on clot microstructure formation producing clots that are more open and porous compared with those formed in its absence. ${ }^{26}$ As a result, we would expect the group with the highest percentage of statin use to have the lowest value of $d_{f}$, however, that is not the case. In the present study, the obstructive CAD group has around $80 \%$ (compared with $65 \%$ in the unobstructive CAD group) of patients taking statin while also having the highest value of $d_{f}$. When comparing statin use and $d_{f}$ for all patients recruited into the study, we found no significant difference between those taking and those not taking statins $(1.736+0.055$ vs $1.741+0.049, \mathrm{p}=0.54)$. A possible reason being the wide range of different types of statin being used in the patients in this study, which may have differing effects alongside the varying comorbidities and heterogeneous nature of the patient population.

A significant increase in $d_{f}$ was also observed in smokers compared with non-smokers/ex-smokers $(1.761 \pm 0.037$ vs $1.733 \pm 0.055, \mathrm{p}=0.001)$. While the number of smokers and non/ex-smokers was not significantly different between the obstructive and unobstructive CAD groups, smoking has been suggested to contribute to the formation of hypercoagulable conditions which could account for its relationship with $\mathrm{d}_{\mathrm{f}}{ }^{27}$

In this study, we found that antiplatelet use was not significantly different between the obstructive and unobstructive CAD groups ( $78 \%$ vs $66 \%, \mathrm{p}=0.11)$. We found 
that when comparing effective antiplatelet (either mono or dual) therapy against non-effective/no antiplatelet therapy, with respect to $d_{f}$, patients with effective antiplatelet therapy had a non-significant decrease in the value of $\mathrm{d}_{\mathrm{f}}(1.735 \pm 0.053$ vs $1.746 \pm 0.053, \mathrm{p}=0.11)$. We have previously shown that mono-antiplatelet therapy with aspirin can lower $d_{f}$ in patients with acute vascular disease, however, this was in a dose of $300 \mathrm{mg}$ of aspirin, not $75 \mathrm{mg}$, and the effect was only an acute lowering of $d_{f}$ which returned to baseline levels after 24 hours. ${ }^{11}$ Despite the fact that monotherapy did not significantly reduce the value of $d_{f}$ in the present study, we did find a significant positive correlation $(0.157, \mathrm{p}=0.014)$ between $\mathrm{d}_{\mathrm{f}}$ and the ASPI test (Multiplate) results showing that the stronger the inhibition of the aspirin the lower the value of $d_{f^{\prime}}$ Of those patients receiving antiplatelet therapy, $12 \%$ were receiving dual antiplatelet. We found a significantly reduced value of $d_{f}$ in those receiving dual antiplatelet therapy compared with no therapy $(1.722 \pm 0.053$ vs $1.746 \pm 0.053, \mathrm{p}=0.048)$. These results suggest that dual antiplatelet therapy is effective in mediating clot microstructure, where patients have lower values of $d_{f}$ corresponding to the production of less densely packed and more porous clots. ${ }^{4}$ This study provides further evidence of the potential of $d_{\mathrm{f}}$ in monitoring the effects of antiplatelet therapy on clot microstructure formation. ${ }^{11}$

The limitations of this study include discrepancy in the numbers collected for both groups. As the study was designed to collect consecutive patients admitted for a routine diagnostic angiography, it was not possible to collect matched patients for both groups. This has resulted in twice as many patients with non-obstructive CAD being recruited ( $n=168$ vs $n=83$ ). While this reduces the power of any statistical analysis, the numbers recruited are still sufficient for meaningful interpretation of the results. Finally, the presence of a $50 \%$ stenosis on invasive coronary angiography was used to define the presence of obstructive CAD. This is an accepted gold standard with prognostic implications. However, intravascular imaging was not used routinely so it is possible that some of the patients with angiographic irregularities or lesions $<50 \%$ may have contained positively remodelled plaques with potentially vulnerable morphology. It is not clear how the presence of these plaques might effect $d_{f}$ and requires further study.

In this study, we have performed the first characterisation of clot microstructure in patients with stable CAD. We have shown that in patients investigated for stable chest pain, the presence of obstructive CAD is associated with an increased potential to produce denser more compact clots and unfavourable morphology. We have also identified that men with obstructive $\mathrm{CAD}$ disease produce unfavourable clot microstructures compared with women. Additionally, patients with CAD receiving dual antiplatelet therapy have a reduced $d_{f}$ value linked to a more favourable looser less compact clot. The $d_{f}$ measurement can potentially be used to risk-stratify patients with stable CAD and has the potential to assess the efficacy of therapeutic interventions by measuring changes in clot microstructure.

Acknowledgements We would like to thank all the staff working in the Haemostasis Biomedical Research Unit, Cardiac Short Stay Unit and Catheterization Laboratory in Morriston Hospital and Princess of Wales Hospital.

Contributors AS and MJL contributed to concept and design, performed rheological analysis, analysed data and critical writing. RA, KH and GRD recruited participants, collected and analysed data, and performed rheological experiments, DO, DS, AC and PT performed angiographies, analysed and collected data and contributed to the writing of the article. KM was the statistician of the project and contributed to concept and design and interpreted data. PRW and PAE contributed to concept and design, revised intellectual content and final approval of the version to be published. There is no additional data available for sharing.

Funding This translational work was supported by the National Institute of Social Care and Health Research (NISCHR) Biomedical Research Unit grant (BR01) and the EPSRC grant (EP/L024799/1).

Competing interests None declared.

Patient consent No identifiable data were used or were reported in the manuscript. All data were anonymised to the researchers.

Ethics approval Wales REC 7 .

Provenance and peer review Not commissioned; externally peer reviewed.

Data sharing statement There is no additional data available for sharing.

Open Access This is an Open Access article distributed in accordance with the terms of the Creative Commons Attribution (CC BY 4.0) license, which permits others to distribute, remix, adapt and build upon this work, for commercial use, provided the original work is properly cited. See: http://creativecommons.org/licenses/by/4.0/

(c) Article author(s) (or their employer(s) unless otherwise stated in the text of the article) 2017. All rights reserved. No commercial use is permitted unless otherwise expressly granted.

\section{REFERENCES}

1. Song CJ, Nakagomi A, Chandar S, et al. C-reactive protein contributes to the hypercoagulable state in coronary artery disease. $J$ Thromb Haemost 2006;4:98-106.

2. Tantry US, Bliden KP, Suarez TA, et al. Hypercoagulability, platelet function, inflammation and coronary artery disease acuity: results of the thrombotic RIsk progression (TRIP) study. Platelets 2010;21:360-7.

3. Reddel CJ, Curnow JL, Voitl J, et al. Detection of hypofibrinolysis in stable coronary artery disease using the overall haemostatic potential assay. Thromb Res 2013;131:457-62.

4. Bratseth V, Pettersen Å, Opstad TB, et al. Markers of hypercoagulability in CAD patients. effects of single aspirin and clopidogrel treatment. Thromb J 2012;10:12.

5. Weisel JW, Litvinov RI. Mechanisms of fibrin polymerization and clinical implications. Blood 2013;121:1712-9.

6. Weisel JW. The mechanical properties of fibrin for basic scientists and clinicians. Biophys Chem 2004;112:267-76.

7. Collet JP, Park D, Lesty C, et al. Influence of fibrin network conformation and fibrin fiber diameter on fibrinolysis speed: dynamic and structural approaches by confocal microscopy. Arterioscler Thromb Vasc Biol 2000;20:1354-61.

8. Mills JD, Ariëns RA, Mansfield MW, et al. Altered fibrin clot structure in the healthy relatives of patients with premature coronary artery disease. Circulation 2002;106:1938-42.

9. Evans PA, Hawkins K, Morris RH, et al. Gel point and fractal microstructure of incipient blood clots are significant new markers of hemostasis for healthy and anticoagulated blood. Blood 2010;116:3341-6.

10. Davies NA, Harrison NK, Morris RH, et al. Fractal dimension (df) as a new structural biomarker of clot microstructure in different stages of lung cancer. Thromb Haemost 2015;114:1251-9.

11. Stanford SN, Sabra A, D'Silva L, et al. The changes in clot microstructure in patients with ischaemic stroke and the effects of therapeutic intervention: a prospective observational study. BMC Neurol 2015;15:35.

12. Lawrence MJ, Sabra A, Mills G, et al. A new biomarker quantifies differences in clot microstructure in patients with venous thromboembolism. Br J Haematol 2015;168:571-5.

13. Davies GR, Pillai S, Lawrence M, et al. The effect of Sepsis and its inflammatory response on mechanical clot characteristics: 
a prospective observational study. Intensive Care Med 2016;42:1990-8.

14. Lawrence MJ, Sabra A, Thomas P, et al. Fractal dimension: a novel clot microstructure biomarker use in ST elevation myocardial infarction patients. Atherosclerosis 2015;240:402-7.

15. Lawrence MJ, Kumar S, Hawkins K, et al. A new structural biomarker that quantifies and predicts changes in clot strength and quality in a model of progressive haemodilution. Thromb Res 2014:134:488-94

16. Al-Azzam SI, Alzoubi KH, Khabour O, et al. The prevalence and factors associated with aspirin resistance in patients premedicated with aspirin. Acta Cardiol 2012;67:445-8.

17. Sibbing D, Braun S, Morath $T$, et al. Platelet reactivity after clopidogrel treatment assessed with point-of-care analysis and early drug-eluting stent thrombosis. J Am Coll Cardiol 2009;53:849-56.

18. Brown MR, Curtis DJ, Rees $\mathrm{P}$, et al. Fractal discrimination of random fractal aggregates and its application in biomarker analysis for blood coagulation. Chaos, Solitons \& Fractals 2012;45:1025-32.

19. Curtis DJ, Brown MR, Hawkins K, et al. Rheometrical and molecular dynamics simulation studies of incipient clot formation in fibrinthrombin gels: an activation limited aggregation approach. $J$ Nonnewton Fluid Mech 2011;166:932-8.
20. Chan MY, Andreotti F, Becker RC. Hypercoagulable states in cardiovascular disease. Circulation 2008;118:2286-97.

21. Mozaffarian D, Benjamin EJ, Go AS, et al. Heart Disease and Stroke Statistics-2016 Update. Circulation 2016;133:e38-e360.

22. Drenos F, Miller GJ, Humphries SE. Increase of plasma fibrinogen levels and variability with age in a sample of middle aged healthy men. Ann Hum Genet 2007;71:43-53.

23. Ernst E, Koenig W. Fibrinogen and cardiovascular risk. Vasc Med 1997;2:115-25.

24. Pedro-Botet J, Climent E, Chillarón JJ, et al. Statins for primary cardiovascular prevention in the elderly. J Geriatr Cardiol 2015;12:431-8

25. Navar-Boggan AM, Peterson ED, D'Agostino RB, et al. Using ageand sex-specific risk thresholds to guide statin therapy: one size may not fit all. J Am Coll Cardiol 2015;65:1633-9.

26. Undas A, Celinska-Löwenhoff M, Löwenhoff T, et al. Statins, fenofibrate, and quinapril increase clot permeability and enhance fibrinolysis in patients with coronary artery disease. J Thromb Haemost 2006;4:1029-36.

27. Nielsen VG, Hafner DT, Steinbrenner EB. Tobacco smoke-induced hypercoagulation in human plasma: role of carbon monoxide. Blood Coagul Fibrinolysis 2013;24:405-10. 